\title{
Chronic hepatitis $C$ in children is a mild and curable liver disease
}

\author{
Vito Di Marco* \\ Gastroenterology and Hepatology Unit, Internal Medicine Department, University of Palermo, Palermo, Italy
}

\section{Hepatitis $C$ in children}

Hepatitis C virus (HCV) infection is a major health problem worldwide, but children constitute only a small portion of infected patients. HCV transmission can occur through blood transfusion, and the incidence of HCV infection was very high in children transfused before 1990 for treatment of chronic haematological diseases [1] or other diseases that required blood transfusion [2]. Since the introduction of screening for HCV infection, blood transfusions have become safe in developed countries, and the residual risk is today very limited.

In developed countries, maternal-fetal transmission has become the major risk factor for HCV in children, with an estimated risk of infection ranging from $2 \%$ to $6 \%$ [3]. The key questions to managing children with HCV chronic infection are identifying the available methods for the staging of the liver fibrosis in children with chronic HCV infection, assessing the risk of developing cirrhosis, and understanding the therapeutic options for curing HCV chronic hepatitis.

Percutaneous liver biopsy is still the standard method for defining the grade of liver inflammation, and the stage of fibrosis. The use of liver biopsy in children is limited by the risk of complications, and by the reluctance, from both children and their parents, to consent to this procedure. However, in the last year, two studies have been published that included children with chronic HCV infection who had undergone liver biopsy before starting antiviral treatment. Both reported a very low percentage of children with significant liver fibrosis. In the study by Sokal et al. [4], more than $85 \%$ of 65 children or adolescents included in a trial of treatment with peginterferon alpha and ribavirin had no fibrosis, or had mild fibrosis; none of them had severe fibrosis or cirrhosis. Another multicentre, controlled trial [5] reported that only 3 out of 104 children evaluated for antiviral treatment had histological evidence of portal fibrosis with septa. Recently, non-invasive methods for assessing liver fibrosis, in place of biopsy, have been developed. Amongst these methods, transient elastography has been applied and studied, mainly in adult patients with chronic HCV. There are few data on stiffness measurement of the liver by FibroScan in children. A study performed on 116 children, with a mean age of 10 years, con-

\footnotetext{
* Tel.: +39 091 6552106; fax: +390916552156.

E-mail address: vito.dimarco@tin.it
}

cluded that liver stiffness measurement is feasible in children, and is correlated with liver fibrosis. The FibroScan equipped with a specific probe could become a useful tool for managing chronic liver diseases in the paediatric population [6].

There are few data regarding the natural history of HCV in children. Nonetheless, in children infected by vertical transmission, the spontaneous clearance of HCV is more common, the risk of fulminant hepatitis is very low, and progression to cirrhosis is less likely than in adults. The rates of spontaneous HCV-RNA clearance reported in the literature for vertically infected children vary considerably. In a retrospective-prospective, Italian cohort study that included $504 \mathrm{HCV}$ infected children [7], the rate of HCV clearance over 10 years of follow-up was around $10 \%$. When the infection became chronic, with persistent positive serum HCV-RNA, liver disease was usually asymptomatic, and the pattern of serum ALT varied; but approximately half of the patients had normal or mildly elevated ALT values. So, it seems clear that children with chronic $\mathrm{HCV}$ infection have a mild disease, which in the first two decades of life rarely evolves towards severe fibrosis or cirrhosis. Though during adolescence, and in adult age, the liver damage can be accelerated by such co-factors as obesity and excessive alcohol consumption.

\section{Antiviral treatment}

For some time, interferon alpha (IFN) was the only treatment available for children with chronic HCV hepatitis. Recently, combination therapy with peginterferon alpha and ribavirin has had positive results in children, as well as in adults. Consequently, regulatory authorities have approved the use of this combination treatment in children. Two randomized controlled trials [4,5] reported a sustained virological response of more than $50 \%$ in children infected with genotype $1 \mathrm{~b}$, and of more than $90 \%$ in patients infected with genotype 2 or 3 . Though there are no reports of life-threatening adverse events in controlled trials, several children have experienced mild or moderate adverse events, and some children have required a reduction in the drug dose, or a discontinuation of treatment. Today, combination therapy of peginterferon alpha and ribavirin is the standard of care, even for children with HCV chronic hepatitis, though the impact of this treatment on clinical practice is still unclear.

In this issue of Digestive and Liver Disease Bortolotti et al. report data on a large cohort of children with chronic HCV hepatitis, and 
assess the impact of therapy on the outcome of the disease [8]. The article has clear clinical relevance, and a number of strengths, despite certain limitations and weaknesses. The main limit of the study is its retrospective design. In any case, this limit is counterpoised by the small number of specialized centres participating in the study, and the high number of children observed in each centre. The second limit concerns the small number of children who underwent liver biopsy, though the available biopsies done in the treated children show mild or no fibrosis in more than $80 \%$ of cases. These data confirm that children generally have mild liver disease.

The study is also worthy of note because it presents the principal epidemiologic and clinical features observed in children in the two decades that followed discovery of HCV, in 1989. More than half of the patients observed in the five Italian centres were born of an infected mother, and most were asymptomatic, with low ALT levels. In addition, genotype 1 was the most frequent genotype detected. These epidemiological, virological and clinical features were comparable with those observed in other recent controlled trials $[4,5]$. The 376 children in this cohort were followed for more than 10 years, and $23 \%$ of them were treated with IFN monotherapy, or with the combination of peginterferon alpha and ribavirin.

\section{Outcomes in paediatric patients}

One of the principal strengths of this study was the virological and clinical outcomes observed in untreated patients during follow-up. Untreated patients had a significantly lower mean age, and also included babies below the age of three. As reported in another study [7], a spontaneous clearance of HCV-RNA was observed in very few children of this group within the fifth year of life. The viral clearance was more frequent in children infected with genotype 3, and was followed by stable ALT normalization in all the patients. Contrastingly, only six children with consistently high ALT levels developed clinical cirrhosis in the period of observation, which was a mean of 10 years.

Of more interest is the data on the efficacy and safety of IFNbased therapy. Before 2000, IFN monotherapy was administrated to 73 children, and 18 (25\%) of them achieved a sustained virological response. Since 2000, 13 children have received a combination therapy with pegIFN alpha-2b and ribavirin, and 12 (92\%) have achieved sustained virological response. The rate of sustained virological response is clearly higher in patients treated with combination therapy than in those treated with monotherapy, even if the comparison between two groups that were treated in different periods, and without randomization, is questionable. Children with genotype 2 or genotype 3 showed a rate of sustained virological response higher than children with genotype 1 . In the latter group, only 1 patient out of 4 achieved a sustained virological response, and this low rate of response could also be influenced by IL28B polymorphisms. In the near future, increased understanding of host genetic factors could help build a model of prediction of the response to therapy in children. It is still too early to know if the new HCV direct-acting antivirals (DAAs) will also be used in children.

Finally, the authors reported adverse events observed during monotherapy and combination therapy. Mild adverse events were experienced by most of the treated children, but only 9 patients (10\%) discontinued therapy because of moderate adverse events or abnormal laboratory values. As reported in other studies, none of the treated children experienced life-threatening complications.

The results of this study seem to confirm that there are three principal clinical patterns that emerge in the natural history of untreated children with hepatitis $C$ infection: the stable clearance of serum HCV-RNA observed early in life in a minority of children; persistent HCV-RNA replication, with mild or moderate liver damage in the majority of patients; and the progression to cirrhosis in a small number of viraemic children with persistently abnormal ALT levels.

Even if the majority of children with chronic infection have mild or moderate liver damage, the efficacy and the acceptable safety profile of combination therapy with peginterferon alpha and ribavirin offer real chances for a cure. Looking at this study, and at those published in the last year, one can conclude that chronic hepatitis $C$ in children is both a mild, and curable disease.

\section{Conflict of interest}

None.

\section{References}

[1] Di Marco V, Capra M, Angelucci E, et al. Management of chronic viral hepatitis in patients with thalassemia: recommendations from an international panel. Blood 2010;116:2875-83.

[2] Vogt M, Lang T, Frosner G, et al. Prevalence and clinical outcome of HCV infection in children who underwent cardiac surgery before the implementation of blooddonor screening. N Engl J Med 1999;341:866-70.

[3] Conte D, Fraquelli M, Prati D, et al. Prevalence and clinical course of chronic hepatitis $\mathrm{C}$ virus (HCV) infection and rate of $\mathrm{HCV}$ vertical transmission in a cohort of 15,250 pregnant women. Hepatology 2000;31:751-5.

[4] Sokal EM, Bourgois A, Stéphenne X, et al. Peginterferon alfa-2a plus ribavirin for chronic hepatitis $C$ virus infection in children and adolescents. J Hepatol 2010;52:827-31.

[5] Wirth S, Ribes Koninckx C, Calzado MA, et al. High sustained virologic response rates in children with chronic hepatitis $C$ receiving peginterferon alfa-2b plus ribavirin. J Hepatol 2010;52:501-7.

[6] de Lédinghen V, Le Bail B, Rebouissoux L, et al. Liver stiffness measurement in children using FibroScan: feasibility study and comparison with Fibrotest, aspartate transaminase to platelets ratio index, and liver biopsy. J Pediatr Gastroenterol Nutr 2007;45:443-50.

[7] Bortolotti F, Verucchi G, Cammà C, et al. Long-term course of chronic hepatitis $\mathrm{C}$ in children: from viral clearance to end-stage liver disease. Gastroenterology 2008;134:1900-7.

[8] Bortolotti F, Indolfi G, Zancan L, et al. Management of chronic hepatitis C in childhood: the impact of therapy in the clinical practice during the first 2 decades. Dig Liver Dis 2011;43:325-9. 